\title{
Sonoelectrochemistry - The application of ultrasound to electrochemical systems
}

\author{
David J. Walton
}

School of Science and the Environment, Coventry University, Priory Street, Coventry CV1 5FB, $U K$

E-mail: $\underline{\text { APX064@coventry.ac.uk }}$

This paper is dedicated to Douglas Lloyd on the occasion of his eightieth birthday

\begin{abstract}
This paper comprises the text of a review lecture on the subject of the effects of ultrasound upon mass transport, upon electrode surface phenomena, upon the behaviour of species and upon reaction mechanisms, and selected examples of the benefits of insonation in electroanalysis, electrosynthesis, electrodeposition and electrochemiluminescence are discussed.
\end{abstract}
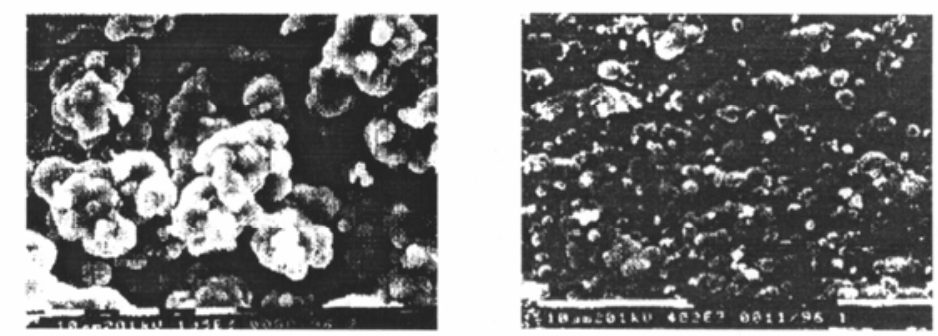

Effect of ultrasound $(800 \mathrm{kHz})$ on polypyrrole electrodeposition lefthand figure silent (SEM 1000x), righthand insonated (SEM 4000x).

Keywords: Sonoelectrochemistry, ultrasound, acoustics, electrosynthesis, electroanalysis, voltammetry, conducting polymers, electrodeposition, cavitation, reaction mechanisms

\section{Introduction}

Electrochemistry is an old discipline. Faraday worked through the 1830's, and Kolbe reported his electroorganic synthetic reaction in $1849 .{ }^{1}$ The vibration of electrochemical cells is likewise a concept of some standing, and Moriguchi reported the improvement of water electrolysis by insonation in $1934 .^{2}$. Thereafter, sporadic reports can be found on sonoelectrochemistry, although this term was not used as a descriptor until more recently. This period included an extensive study by Yeager upon the physicochemical parameters of ionic solutions. ${ }^{3}$ Ultrasound 
was also shown to affect metal electrodeposition with benefit to the quality of the deposit, its adhesion and morphology, and also the diminution of brighteners and other additives needed in silent systems, such as the toxic molecule thiourea. ${ }^{4}$ Other effects of ultrasound on phase phenomena included emulsification to facilitate the electrolytic dechlorination of polychlorinated aromatic molecules. ${ }^{5}$ Ultrasound (which can affect per se the RMM of polymers ${ }^{6}$ ) also influenced a series of electroinitiated polymerizations, ${ }^{7}$ and separately had been shown to affect the electrogeneration of organochalcogenide species. ${ }^{8}$

At this point, in the late 1980s, we produced our first paper from Coventry showing a mechanistic switch in an electroorganic synthetic reaction, namely the Kolbe electrooxidation of cyclohexanecarboxylate in methanol. Here the preponderance of the one-electron per molecule dimer bicyclohexyl in the silent system was converted into two-electron per molecule products such as cyclohexyl methyl ether and cyclohexene in the insonated system. ${ }^{9}$ Our paper closely followed one reporting the effect of ultrasound on electrodeposition of the conducting polymer polythiophene, ${ }^{10}$ and somewhat predated the first in a series of reports on various organic sonoelectrochemical systems by Nonaka, Atobe et al. ${ }^{11}$ These workers found a reverse mechanistic switch in the electroreduction of carbonyl compounds, from the two-electron-permolecule monomeric alcohol in the silent system, to the one-electron per molecule dimeric pinacol under insonation.

Since then, interest in sonoelectrochemistry has grown considerably, with some 100 papers published last year using this keyword. This is a result of the general enhanceement in both electrochemical and sonochemical equipment and methodology, allowing a wider range of experiments and a greater understanding of phenomena. Comprehensive studies have been made by several groups including Nonaka and Atobe at Tokyo, and particularly by RG Compton et al. at Oxford, who have produced a very significant proportion of recent publications in the field. There have been a number of reviews ${ }^{12-16}$ to which the reader is directed for a more thorough account of the history and of recent developments.

It must also be noted that while the underpinning funding for these studies has come from a variety of sources, including National Research Councils and their equivalents, the considerable European effort into sonoelectrochemistry is due to specific support by the European Union under a series of overlapping initiatives into 'Non-Classical Methodologies' in chemistry. Thus, Coventry, Oxford, Brussels and Coimbra Universities collaborated in a Human Capital and Mobility Network in sonoelectrochemistry as part of the EU Fourth Framework initiative, which included the COST D6 programme 'Chemical Reactions and Processes Under Extreme and NonClassic Conditions', and currently Coventry, Oxford, Brussels, Coimbra, Alicante and Rostock Universities comprise a Fifth Framework COST D10 Working Group on sonoelectrochemistry in the programme 'Innovative Methods and Techniques for Chemical Transformations' ${ }^{17}$

Sound comprises longitudinal waves that cause compression and rarefaction cycles as they passes through a medium. Audible sound covers the frequency range from approximately $16 \mathrm{~Hz}$ to $16 \mathrm{kHz}$, and ultrasonic frequencies are higher than this. Generally the frequency region just above hearing is called 'power' ultrasound $(20-100 \mathrm{kHz})$, and this where ultrasonic cleaning 
baths and commercial plastic welding apparatus operate, while frequencies near and above 1 $\mathrm{MHz}$ are used for diagnostics, medical scans and other analytical applications. The effects of ultrasound in a liquid are to cause 'acoustic streaming' and/or the formation of cavitation bubbles, depending upon the parameters of ultrasonic power, frequency, sonic source characteristics, and solution phenomena such as viscosity, volatility, and the presence of dissolved gases or other nucleation sites. ${ }^{18}$ Calculations have shown that the formation and decay of cavitation bubbles involve transient extremes of temperature and pressure, and the bubbles can form microjets that impinge towards a surface. These are responsible for the cleaning effect of a laboratory ultrasonic cleaning bath and the obervation of neat holes punched through a sheet of aluminium cooking foil held vertically in such a bath. However, other phenomena occur, and sonoluminescence, the weak evolution of light particularly from insonated aqueous systems, is of fundamental physicochemical significance. ${ }^{19}$ The placing of a heterogenous solid interface, the electrode surface, into a liquid medium under insonation offers a number of potential effects, particularly given the range of electrochemical parameters that may be varied in addition to ultrasonic ones. Thus, solvent, electrolyte, electrode material, cell geometry and geometric disposition of the electrode, electrode potential and/or dynamic modulation of potential or current density (for example by pulsing or cyclic scanning), and the presence of quenching or trapping agents, may all be varied within a single system to affect the course of electrochemical reaction of the substrate molecule. This gives a great deal of experimental flexibility in sonoelectrochemical methodology.

A number of possible effects of ultrasound upon an electrochemical system may be predicted:

1. A general improvement of hydrodynamics and movement of species;

2. The alteration of concentration gradients at various points in the reaction profile, and consequent switching of kinetic regimes with effect on mechanism and reaction products;

3. A cleaning and abrading effect upon an electrode surface, thus obviating fouling problems, or else altering the nature of coatings that manage to form;

4. Sonochemically-induced reactions of intermediate species that have been generated electrochemically;

5. The sonochemical formation of species that react electrochemically in conditions where the silent system is electroinactive.

These possibilities are now discussed using results from Coventry and elsewhere.

\section{Sonovoltammetry}

A current/voltage plot for a simple reversible couple, such as ferricyanide in water or ferrocene in acetonitrile can have two general shapes, depending upon a number of factors. In particular differential diffusion characteristics due to the geometric area and shape of the electrode have an effect. At very small electrodes ('microelectrodes') the silent system shows an eventual flat plateau, the 'limiting current', while at larger electrodes ('millielectrodes') the shape is a 
characteristic smooth peak in each scan direction, as shown in Figure 1. Note the different current values, reflecting the different electrode areas. ${ }^{20}$

The same electrode systems, respectively, are shown exposed to ultrasound in Figure 2. Not only has the measurable current for both sizes of electrode increased by an order of magnitude, with benefit for the viable detection range of an electrochemical sensor, but the

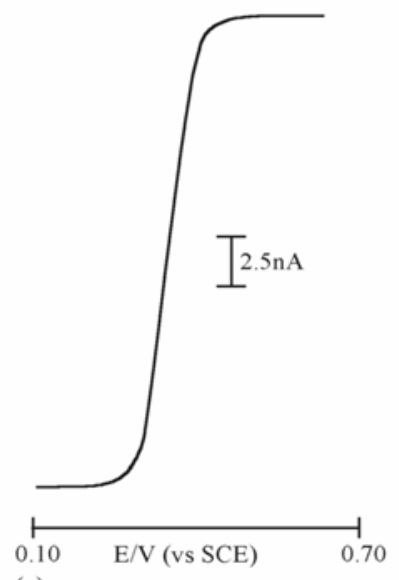

(a)

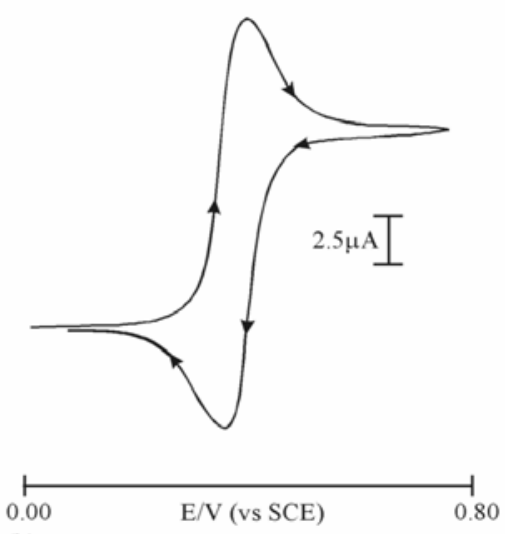

(b)

Figure 1. Silent Voltammograms for the oxidation of $\mathrm{Cp}_{2} \mathrm{Fe}$ at platinum in Acetonitrile/TBAP, sweep rate $20 \mathrm{mVs}^{-1}$, trace a) microdisc electrode radius $13.6 \mu \mathrm{m}$; trace b) macrodisc radius $0.1 \mathrm{~cm}(\mathrm{ref} 20)$.

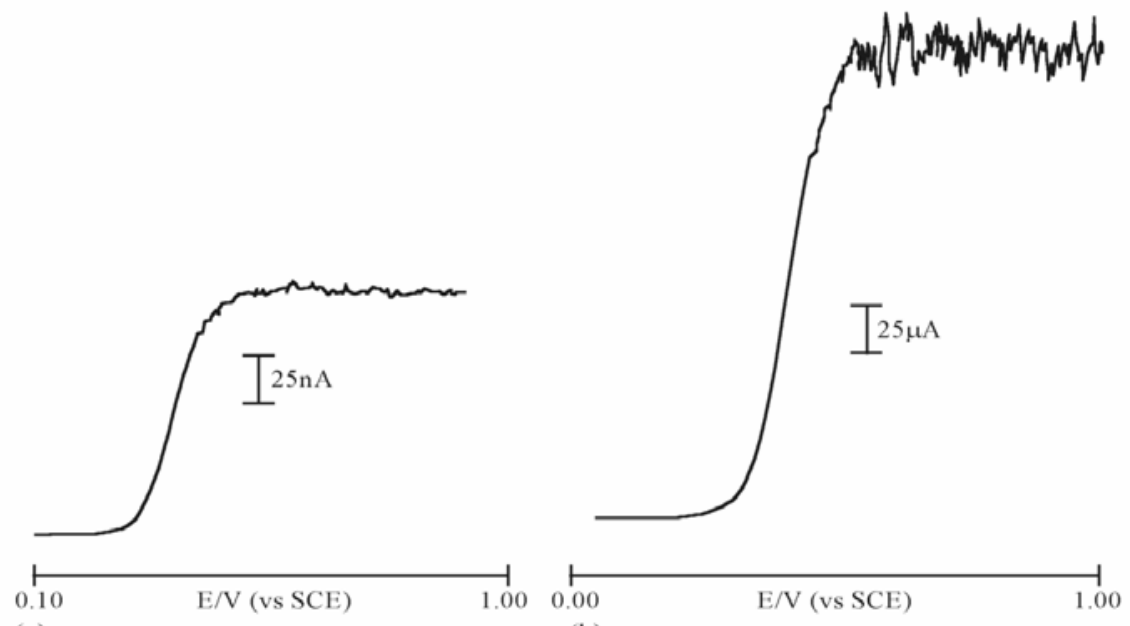

(a)

(b)

Figure 2. Sonovoltammograms for $\mathrm{Cp}_{2} \mathrm{Fe}$ in MeCN/TBAP, conditions as for figure 1 except ultrasound at $20 \mathrm{kHz}$, intensity c. $45 \mathrm{Wcm}^{-2}$. Note enhanced current scales. (from Reference 20). shape of the insonated trace from the millielectrode has a limiting-current plateau typical of that obtained in a silent system from a microelectrode. In essence, an insonated millimeter-scale disc 
or wire electrode now behaves like a silent microelectrode, and this may be useful in the design and assembly of practical sensor systems, for example to be placed in river water, where the small scale of microelectrodes can make them finicky to manipulate by users of the sensor who might not be trained electrochemists.

In electroanalysis rigorous regard for reproducibility favours use of a sonic horn (c.20kHz) as ultrasound source, usually placed face-on at a known (short) distance to, for example, a disc electrode as shown in the electroanalytical cell (Figure 3) developed by Compton and coworkers and now widely used, ${ }^{16}$ although a sideways-on geometry also produces a substantial current increase in the voltammogram, ${ }^{21}$ while even the less well-controlled geometry of a wire electrode in a cell placed simply in an ultrasonic bath can produce a ten-fold current increase. ${ }^{20} \mathrm{~A}$ considerable number of electroanalytical systems have now been studied by a range of sonoelectrochemical protocols. ${ }^{15,16}$ One area that remains unclear, however, is the question of whether ultrasound alters the physicochemical parameters of electron-transfer. This could happen by a number of mechanisms, for example by alteration of the surface of an electrode by affecting oxide layers or adsorption phenomena in a particular reaction system, or else by localized heating of the electrode surface and layers near it due to a mismatch of acoustic impedance at the solid/liquid interface.

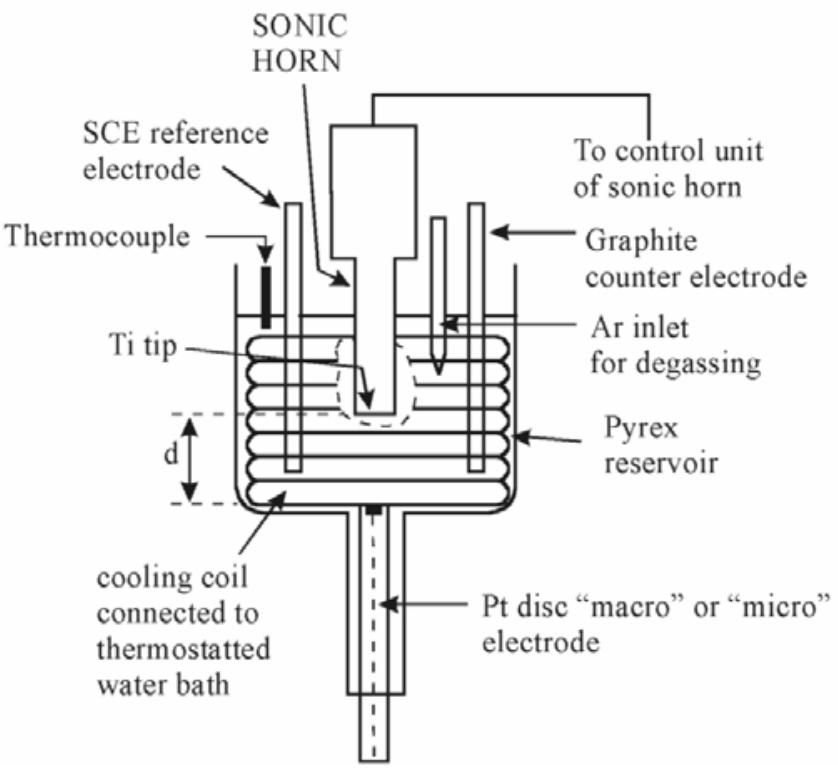

Figure 3. Thermostatted sonoelectrochemical cell with the tip of a sonic horn at a distance $d$ from the electrode surface. Relative positions of the electrode and sonic source can be inverted. ( from ref. 16). 
Strictly the half-wave potential of a system that is reversible in silent conditions ought not to be altered unless reversibility is affected by for example some ultrasonically-induced chemical process, but cavitation bubbles involve extremes of temperature and pressure as they form and decay; current fluctuations due to their impingement on a solid electrode surface can be monitored by fast-transient techniques, ${ }^{22}$ and it may be that equilibrium assumptions normally made for reversible silent systems do not apply to insonated ones. It is notable that insonation at similar sonic powers (within the limits of power-matching - see below) from baths of widelydiffering frequencies ( $25 \mathrm{kHz}$ and $800 \mathrm{kHz}$ ), gives similar increases in limiting current from a reversible couple at a platinum wire electrode, as shown in Figure $4 .{ }^{20}$

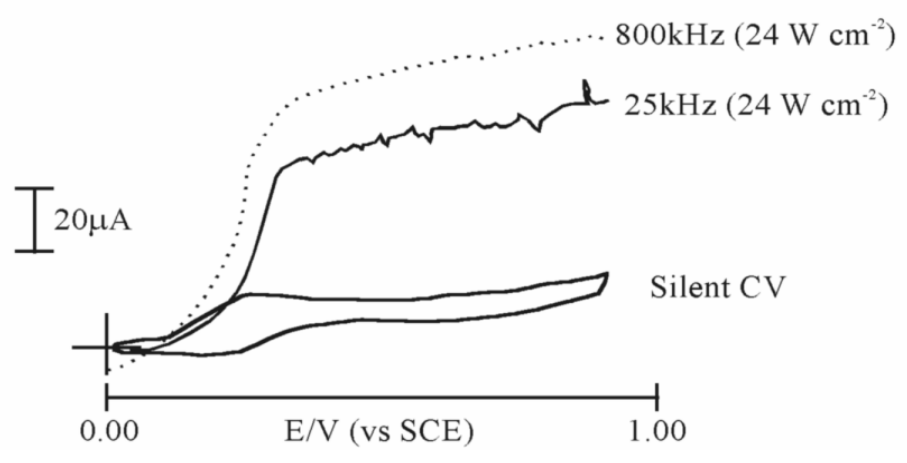

Figure 4. Effect of ultrasonic frequency at nominally fixed powers from $800 \mathrm{kHz}$ bath and $25 \mathrm{kHz}$ probe sources upon ferricyanide voltammetry at a platinum wire (from reference 20).

\section{Sonoelectrosynthesis}

It is fair to say that the majority of reports on the insonation of electrosynthetic reactions have identified some effect on products, yields or process parameters. Many electrosynthetic reactions are in principle multipathway, offering a number of potential mechanisms, although in fixed conditions, such as in a silent system, one pathway may predominate. Figure 5 gives a simplified picture of some possible options for the electroreduction of a neutral starting molecule. Disregarding the mechanisms involving cleavage reactions of intermediates, if protonation can occur then there is a possible one-electron per starting molecule pathway to a hydrodimer, and a two-electron pathway to a dihydro monomeric species. This describes the possibilities for chemical or electrochemical reduction of carbonyl compounds, which can give either a pinacol (in systems that favour radical dimerisation), or a secondary alcohol. The one-electron process can be preferred chemically by use of a mercury amalgam reducing agent, while an ionic reducing agent such as a hydride species favours the secondary alcohol. Nonaka et al. showed that insonation of ketone electroreduction at $36 \mathrm{kHz}$ dramatically switches the product mix from a slight preponderance of 2-electron alcohol (ratio two- to one-electron of 60/40) over to a marked preference for the 1-electron pinacol (ratio two- to one-electron of 23/77), with improved overall current efficiency, ${ }^{11}$ (in this paper the group also showed that a similar 2-electron to 1- 
electron switch occurred for the reduction of benzyl bromide, which involves a cleavage reaction -cf Figure 5).

Nonaka et al. also investigated stereochemical aspects in the formation of pinacols. ${ }^{23}$ By altering the ultrasonic power level, this group later attempted to relate this product switch to the cavitation threshold, at which this phenomenon becomes important in the medium. ${ }^{24}$ An explanation for the mechanistic change to products with fewer electrons-per-molecule in constant-current systems is simple increase in hydrodynamics, where improved mass transport favours dimerisation rather than a second electron transfer.

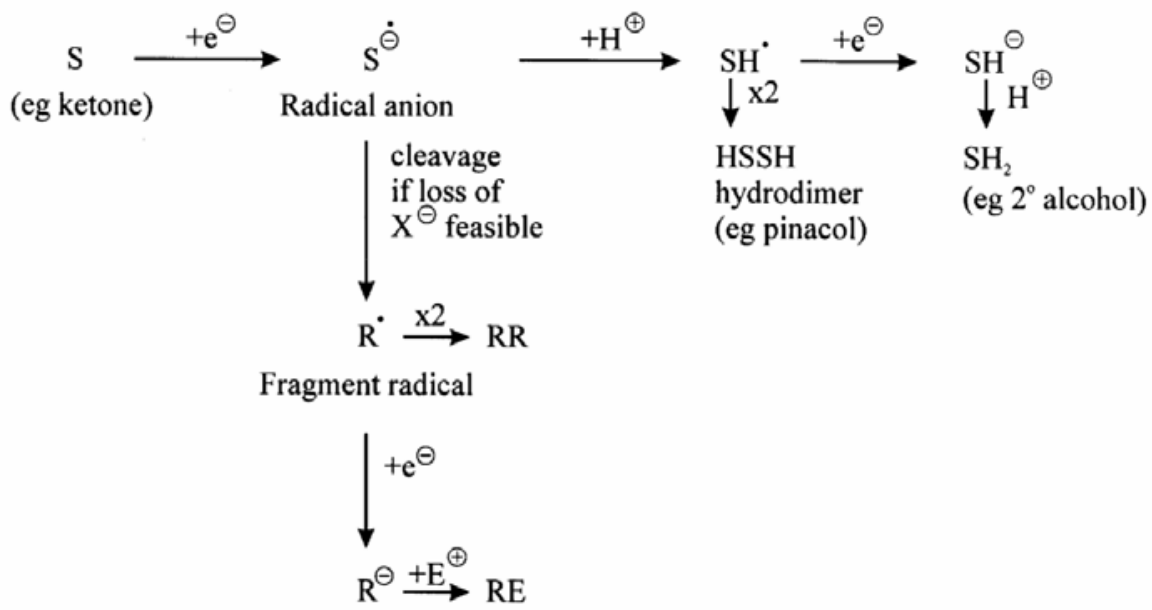

Figure 5. Representative electroreductive pathways for uncharged starting molecule S.

We have also studied ketone reduction for a range of substituted acetophenones, and have confirmed the switch shown by Nonaka. However, in reproducing his experiment we noticed substantive weight loss from the soft lead electrode (chosen because of this metal's high overpotential against the competing hydrogen evolution reaction in aqueous methanol solvent). Nonaka's and our experiments used ultrasound in the 'power' frequency range $(20-50 \mathrm{kHz})$, which is where ultrasonic cleaning baths operate, precisely because of the abrasive and scouring power of cavitation. We therefore tried a focused $800 \mathrm{kHz}$ bath, operating at a higher frequency where cavitation phenomena occur over a much shorter timespan, and bubbles per se cannot be seen by the naked eye. We noticed that there was very little mass loss from the lead electrode at this higher frequency, but the same switch in mechanism occurred, favouring the one-electron pinacol.

The options for pentamethylbenzene electrooxidation are shown in Figure 6A, and can be summarized as a two-electron route to nucleophilic substitution at the alkyl side-group, compared to one-electron dimerisation. However, this may be either by a new carbon-carbon bond linking two pendant alkyl groups, or preferentially by a new bond between one alkyl group 
and the free ring position of the second ring, as shown. There are a number of isomers in this latter situation, which were not distinguished in our analysis. The sequence of steps in the mechanism is not necessarily as shown in the scheme.

A)
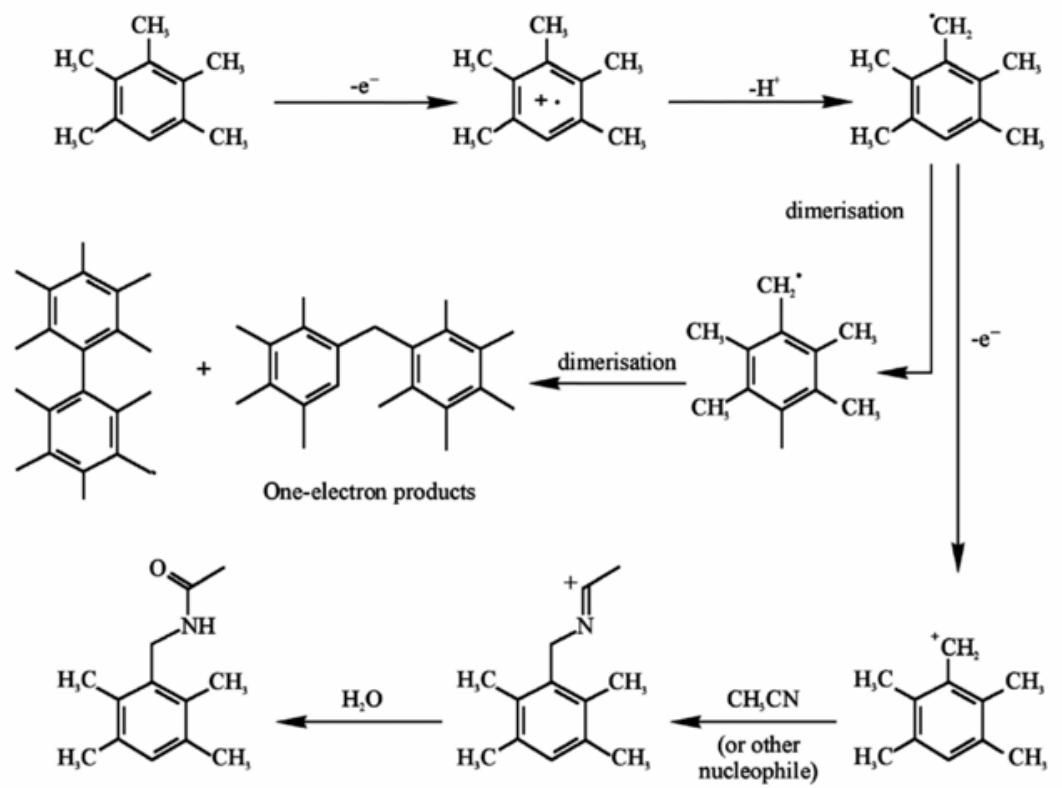

Two-electron products

B) a)<smiles>CC(=O)OCc1c(C)cc(C)c(C)c1C</smiles>

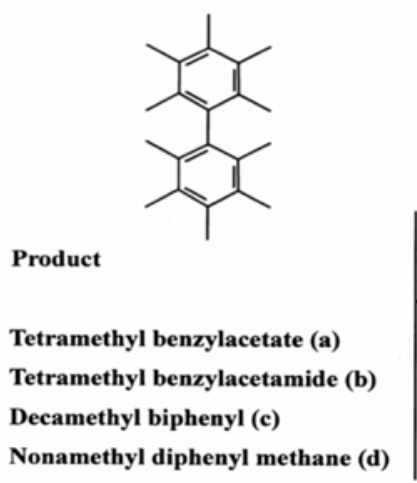

b)<smiles>CC(=O)NCc1c(C)cc(C)c(C)c1C</smiles>

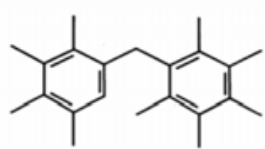

(1) (2) (3)

\begin{tabular}{|c|c|c|}
$\begin{array}{c}\text { mechanical } \\
\text { stirring }\end{array}$ & $\begin{array}{c}\text { ultrasound } \\
38 \mathrm{kHz} \\
\text { bath }\end{array}$ & $\begin{array}{c}\text { ultrasound } \\
800 \mathrm{kHz} \\
\text { bath }\end{array}$ \\
\hline 24 & 11 & 23 \\
42 & 62 & 24 \\
8 & 9 & 7 \\
14 & 14 & 43
\end{tabular}

Figure 6. Electrooxidations of alkylaromatics in the presence of nucleophiles:

6A: Representative pathways for pentamethylbenzene in acetonitrile with aqueous work-up.

6B: Product ratios from pentamethylbenzene in acetonitrile/acetate medium in silent conditions and insonated from $38 \mathrm{kHz}$ and $800 \mathrm{kHz}$ baths - effect of ultrasonic frequency (from reference 36) 
We found a slight shift from two-electron per molecule products to one-electron products under insonation at $38 \mathrm{kHz}$, but a more striking shift under insonation at $800 \mathrm{kHz}$, as shown in Figure 6B. Now a persistent and presently unresolved issue in sonochemistry is the matching of actual powers delivered at different frequencies. It is possible to measure electronic power delivered to a piezoelectric transducer, but since reflection of sound waves and the formation of standing wave patterns varies considerably with the sound wavelength (which is in itself solvent and medium dependent), and since an electrochemical cell placed in a sonic bath is a glass container with electrodes and other solid objects that interfere differently with sound at different frequencies, it is not at all clear that powers are the same for the same electrolysis cell placed in baths that operate at two substantially different frequencies. The accepted way to match sonic powers is by omitting cooling (all sonoelectrochemical systems are cooled during operation because of adventitious heat from insonation) and to measure the initial temperature rise of the cell. We did this, but because of the different geometry of the two ultrasonic baths (at $38 \mathrm{kHz}$ and $800 \mathrm{kHz}$ ) we cannot be sure of power matching in this comparison. However, in practical terms if effective ultrasonic power is a factor responsible for mechanistic discrimination then this is a further benefit in sonoelectrosynthesis. Note that in laboratory-scale electrosynthetic reactions, electrodes of centimeter-scale are required, and the uniform sound field employed in an electroanalytical system using microelectrodes cannot be attained, especially in a divided cell. The interesting thing is that even in these much less well-defined acoustic conditions sonoelectrosynthetic reactions show substantial effects of insonation.

This effect of insonation on electrode fouling is further discussed below. Note that insonation improved the isolated yields of product mixtures, reflecting an overall increase in current efficiency for passage of the same charge. Furthermore in all reactions ultrasound reduced the effective cell potential needed to drive the constant-current electrolyses, with energy saving, at least as regards the electrochemical cell.

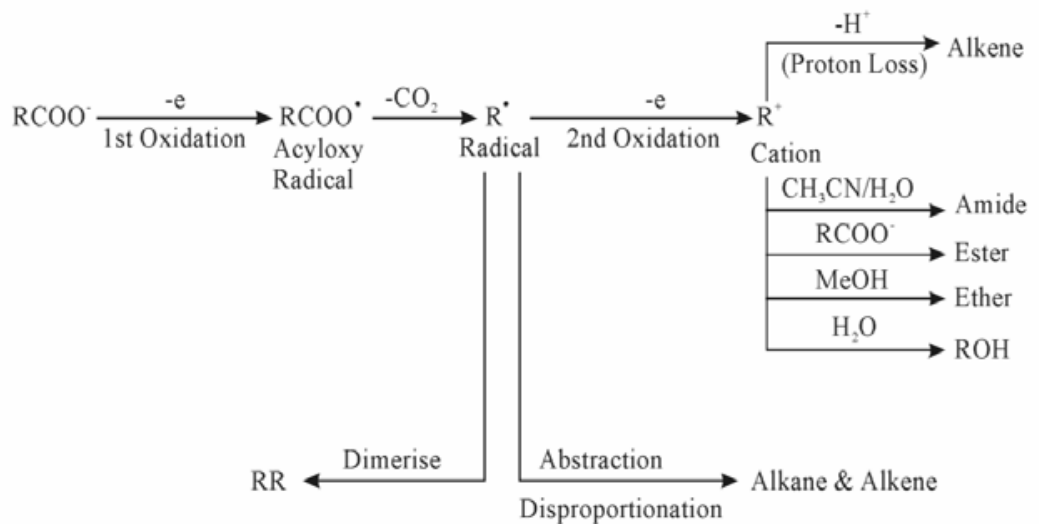

Figure 7. Reaction Pathways for the Electrooxidation of Carboxylates. 
We have also conducted a study into the electrooxidation of carboxylates (the Kolbe Electrooxidation, see Figure 7). Originally we chose a system poised between the one-electron (radical dimerisation to the Kolbe dimer) and two-electron (products via carbocation intermediate) pathways. However, the mechanism is complicated by loss of carbon dioxide at some point in the process. The Kolbe Reaction is particularly prone to mechanistic switching by choice of conditions, including solvent, acidity, electrode material, current density and other factors $^{26}$, and adsorption phenomena are important in aqueous systems. We chose the oxidation of partially-neutralised cyclohexanecarboxylic acid (the carboxylate anion is the reactive species) in dry methanol, where the dimer bicyclohexyl is obtained in similar yield to the total of twoelectron products; and found that on insonation the product mix switched to favour the twoelectron products as shown in Table 1 . This is in contrast to the systems described above where in general insonation favours the route with fewer electrons.

Table 1. Kolbe electrooxidation of cyclohexanecarboxylate at platinum in methanol (taken from ref. 30)

a) product rations under silent conditions and at two insonation frequencies

b) total yields of extracted product mixtures

\begin{tabular}{cccc}
\hline Product & Silent & $38 \mathrm{KHz}$ & $850 \mathrm{KHz}$ \\
\hline Cyclohexane & 7 & 3 & 3 \\
Cyclohexene & 12 & 22 & 29 \\
Cyclohexyl methyl ether & 3 & 31 & 31 \\
Bicyclohexyl & 43 & 11 & 6 \\
Methyl cyclohexanoate & 16 & 14 & 9 \\
Cyclohexanol & 18 & 12 & 21 \\
Other products & 1 & 3 & 1 \\
\hline
\end{tabular}

1a. Product ratios (\% by GC) from cyclohexanoate electrooxidation after passage of $2.0 \mathrm{~F} \mathrm{~mol}^{-1}$, at platinum in methanol

\begin{tabular}{ccccc}
\hline $\begin{array}{c}\text { Current density } \\
/ \mathrm{mA} \mathrm{cm}^{2}\end{array}$ & $\begin{array}{c}\text { \% Partially } \\
\text { neutralised }\end{array}$ & Silent & 38 KHz & $850 \mathrm{KHz}$ \\
\hline 150 & 25 & 0.8 & 1.2 & 1.6 \\
200 & 50 & 0.7 & 1.0 & 2.1 \\
\hline
\end{tabular}

1b. Total ether-extracted quantities (g) from cyclohexanoate electrooxidation (from $4 \mathrm{~g}$ of starting material, after passage of $2.0 \mathrm{~F} \mathrm{~mol}^{-1}$ ).

When partially-neutralised phenylacetic acid was used, which cannot form an alkene as one of the two-electron products, then the product switch was much less pronounced, as shown in Table 
2. Here there is a further processing benefit of ultrasound, because in the silent reaction substantial amounts of pyridine have to be added to the electrolysis to prevent the formation of a film coating on the anode (working electrode) that is deleterious to continuance of electrolysis. This pyridine is not necessary under ultrasound. The electrooxidation of $p$-chlorophenylacetic acid is shown in Table 3. This reaction is known to favour two-electron and higher mechanisms and there is no effective product switch with insonation. However, $p$-chlorophenylacetate electrooxidation in methanol also suffers from unexpected electrode fouling, this time from a coating on the cathode (counter electrode). In both cases sonication at $35 \mathrm{kHz}$ removed the electrode coatings and allowed electrolysis to proceed. ${ }^{27}$

Table 2. Average product distribution from electrochemical oxidation of phenylacetate in absence and presence of ultrasound ${ }^{\mathrm{a}}$ (taken from ref. 27)

\begin{tabular}{cccc}
\hline \multicolumn{4}{c}{ Relative product distribution ${ }^{\mathrm{b}}$} \\
\hline & $\begin{array}{c}\text { 13\% Pyridine } \\
\text { no ultrasound }\end{array}$ & $\begin{array}{c}13 \% \text { Pyridine } \\
\text { ultrasound }\end{array}$ & $\begin{array}{c}\text { No pyridine } \\
\text { ultrasound }\end{array}$ \\
toluene & 0.4 & 1.5 & 3.1 \\
benzyl methyl ether & 21.1 & 27.8 & 32.3 \\
methyl phenylacetate & 10.2 & 4.2 & 6.2 \\
bibenzyl & 59.8 & 51.3 & 52.7 \\
others & 8.3 & 12.4 & 5.7 \\
\hline
\end{tabular}

${ }^{a}$ Average potential difference across the cell for current density $100 \mathrm{~mA} \mathrm{~cm}{ }^{-2}$ is $7.9 \mathrm{~V}$ in the absence and $6.6 \mathrm{~V}$ in the presence of ultrasound.

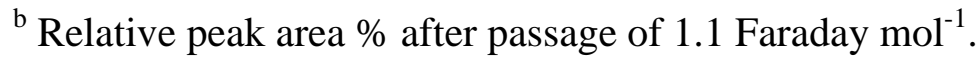

${ }^{\mathrm{c}}$ Without pyridine there is rapid cessation of reaction under silent conditions.

${ }^{\mathrm{d}}$ Powder precipitate, additional product in $14 \%$ weight yield.

Nonaka has also examined Kolbe reactions in aqueous media, without seeing such a mechanistic switch ${ }^{28}$ and more recently Compton et al., studying hydrophobic carboxylates emulsified in aqueous media by ultrasound, and using the physical effect of insonation to influence availability at the electrode in the environmentally-beneficial solvent water, did not find such a favouring of the two-electron products. ${ }^{29}$ However, we have conducted 'kinetic runs' on partially-neutralised cyclohexanecarboxylate in our dry methanol system, and have shown that the first part of the insonated reaction actually shows a brief trend towards one-electron products before a prevailing switch towards the two-electron mechanism takes over. ${ }^{30}$ We also tested the reaction at $850 \mathrm{kHz}$ and found the same overall switch to the two-electron products, with the same brief contradictory initial reaction. Our Kolbe reactions are performed at very high current density (> $100 \mathrm{mAmp} \mathrm{cm}^{-1}$ ) with two centimeter-square platinum foil electrodes only a millimetre or so apart and there is vigorous gassing from the cathode during reaction. It may be that there is a change in local 
reaction conditions during electrolysis that provides the mechanistic time-dependence, but if so it is much less-pronounced in silent (but mechanically-stirred) conditions where there is still the vigorous gassing.

Table 3. Average product distribution from electrochemical oxidation of $p$-chlorophenylacetate in absence and presence of ultrasound ${ }^{a}$ (taken from ref. 27)

\begin{tabular}{lll}
\hline Relative product distribution $^{\mathrm{b}}$ & & \\
\hline & Silent $^{\mathrm{c}}$ & Sonicated $^{\mathrm{d}}$ \\
p-Chlorobenzyl methyl ether & 71.5 & 69.3 \\
Methyl $p$-chlorophenylacetate & 3.4 & 2.5 \\
p-Chlorobenzaldehyde & 9.1 & 4.2 \\
p-Chlorobenzyl alcohol & 3.5 & 9.0 \\
-Chlorobenzaldehyde acetal & 10.8 & 11.2 \\
others & 1.7 & 3.8 \\
\hline
\end{tabular}

${ }^{a}$ Average potential difference across the cell for current density $100 \mathrm{~mA} \mathrm{~cm}{ }^{-2}$ is $5.63 \mathrm{~V}$ in the absence and $4.83 \mathrm{~V}$ in the presence of ultrasound.

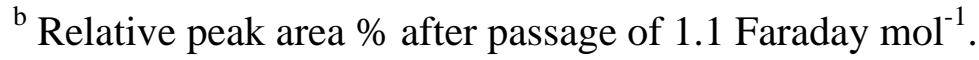

${ }^{\mathrm{c}}$ Total weight yield after extraction - 23\%.

${ }^{\mathrm{d}}$ Total weight yield after extraction $-46 \%$.

Ultrasound is known to roughen metal surfaces, but the mechanistic change does not reflect permanent alteration in the nature of the platinum surface however, because electrodes re-used show the same time-dependence. Another feature of the high-frequency system was the visual clarity of the yellow final reaction mixture, which is normally very dark brown-coloured, both when silent and under $38 \mathrm{kHz}$ insonation. This concurs with a 4-fold improvement in isolated yield of product mixture under $850 \mathrm{kHz}$ insonation, compared to silent conditions, as shown in Table 1. These reactions are not run to completion, and current efficiencies in silent conditions are low, but high-frequency ultrasound produces a substantial improvement in yield as well as a switch in product distribution. This is further shown by the diminution of cyclohexanecarboxylate methyl ester side-product. This is thought to be formed by chemical esterification of methanol solvent with unreacted cyclohexanecarboxylic acid, catalysed by protons produced at the anode, and is a persistent parasitic product in silent conditions. It is substantially reduced under insonation, suggesting that oxidative discharge of the solvent methanol is suppressed.

The above reactions all show that the imposition of ultrasound, with its time-dependent parameters, provides a means of perturbing reaction mechanisms. Many electroorganic mechanisms are complex, and this methodology provides a useful means of elucidating mechanistic discrimination. 


\section{Organic electrodeposition and electrode coatings}

Since ultrasonic cleaning baths are designed to abrade dirt from surfaces immersed in them it is little surprise that one effect of insonation at frequencies around $30 \mathrm{kHz}$ is to keep electrode surfaces clean. This was shown by the electrosynthetic oxidations of phenylacetate and $p$ chlorophenyl-acetate mentioned above. A feature of the phenylacetate system is that a powder is obtained from the solution with ultrasound, which appears to be a predominantly para-linked polymer from linking of benzyl and benzoyl radicals. Trapping of the acyloxyradical in a Kolbe reaction as a straightforward linear compound is unusual, although trapping by internal cyclisation has been reported in an appropriate system such as the electrooxidation of 3,3diphenylacrylate, which gives 4-phenylcoumarin among the products. ${ }^{31}$

Ultrasound also keeps the electrode surface clean, seen in repetitive voltammograms from chromium hexacarbonyl in acetonitrile at platinum, a well-known case of electrode fouling. ${ }^{32}$ We also showed diminution of electrode fouling in the anodic electrochemiluminescence from ruthenium trisbipyridyl in aqueous oxalate solution. Here the electrooxidatively generated $\mathrm{Ru}(\mathrm{III})$ meets oxalate chemical reductant in solution to give excited $\mathrm{Ru}(\mathrm{II})$ that emits light. The anode typically becomes fouled with time, and light emission drops, but insonation allows repetitive usage of the same electrode without loss of emission, as shown in Figure 8. This system also suffers from patchiness and edge effects when centimeter-scale electrodes are used, while millielectrodes that minimize these problems give only low light levels because of their small areas. Ultrasound improves mass transport such that uniform emission occurs across the area of a centimeter-scale electrode, with consequently greater light levels emitted. The trisbipyridyl unit may have for example an amide functionality as shown in Figure 9, so as to allow attachment of the complex to an enzyme for a chemiluminescent bioassay, and the sonicated sytem allows a lower detection limit by using a larger area electrode, and without fouling problems. ${ }^{33,34}$

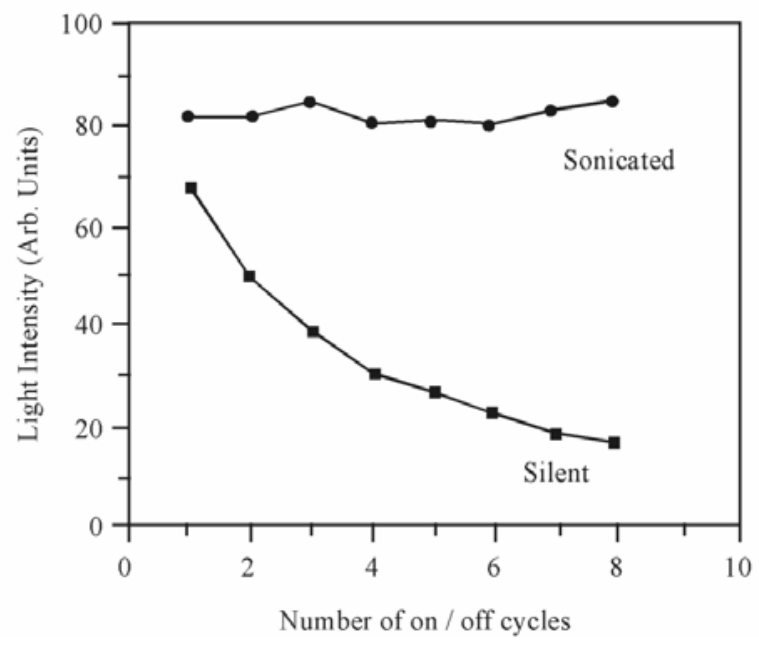

Figure 8. Diminution of electrode fouling during anodic electrochemiluminescence from ruthenium trisbipyridyl in aqueous oxalate (taken from reference 33). 


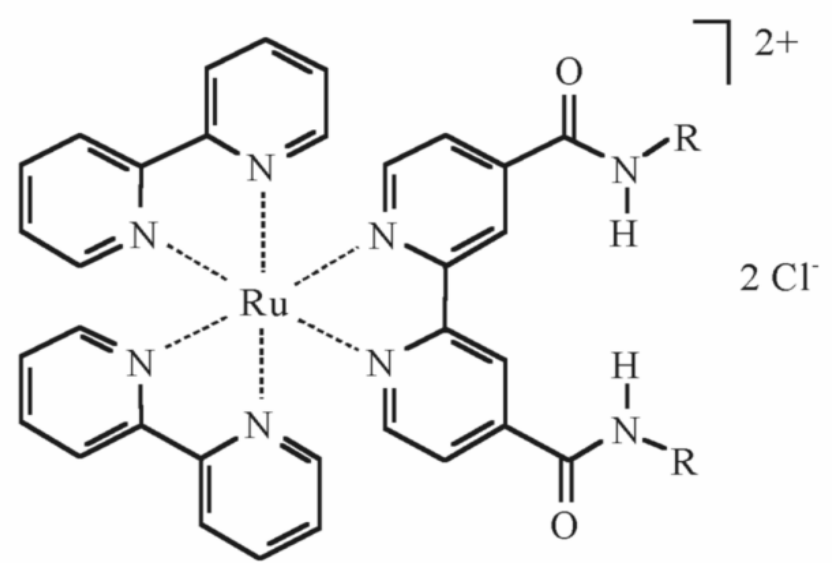

Figure 9. Functionalised ruthenium trisbipyridyl derivative for electrochemiluminescence assays.

However, not all electrode coatings are deleterious, and metal electrodeposition has long been known to be enhanced in various ways by ultrasound. ${ }^{4}$ Conducting polymers of the polypyrrole type may be considered to be electrode coatings, and improved morphology and other enhancements in polythiophene electrodeposition under insonation in the $20-40 \mathrm{kHz}$ frequency range have been shown. ${ }^{10}$ More recently ultrasound has been seen to influence polyaniline electrodeposition. ${ }^{35}$ We found that $20 \mathrm{kHz}$ insonation using a cleaning bath interfered with polypyrrole formation at the electrode, but use of an $850 \mathrm{kHz}$ higher-frequency bath allowed the formation of good polymer films on the electrode. ${ }^{36}$ These films had finer structural features and a denser morphology than silent ones as shown in Figure 10. There is an interesting aspect to this study. Separately we have shown that when polypyrrole is prepared by electrooxidation of the monomer in the presence of more than one anion in the electrolyte then there is a competition between anions such that their ratios in the resulting polymer may not mirror the composition of the solution.

For example equal ionic ratios in an aqueous electrolyte containing perchlorate ion and sulphonate groups from tetrasulphonato copper phthalocyanine produces a polymer substantially-rich in the hydrophobic dye, whereas in acetonitrile, all other parameters remaining the same, the polymer is rich in perchlorate ion. ${ }^{37}$ This switch may be explained on grounds of hydrophobic interactions, since a more hydrophilic sulphonate, $p$-toluene sulphonate does not produce this discrimination when mixed with perchlorate in an aqueous medium, and a nearer-toequal anion composition is found in the polymer. ${ }^{38}$ However, less easy to explain is the pronounced discrimination between two anions of similar size, charge, and solubility properties, perchlorate and hexafluorophosphate. For a range of pyrroles, thiophenes, substituted derivatives of these monomers, and also the carbocyclic molecule azulene (which does not have a ring heteroatom), at a range of electrode materials, and in a range of solvents including water, propylene carbonate, and acetonitrile 


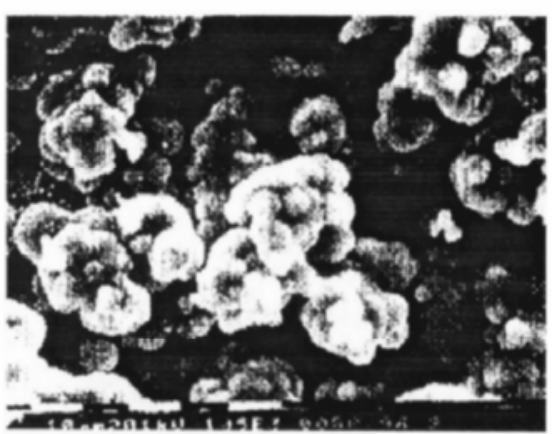

SEM (1000x): non sonicated

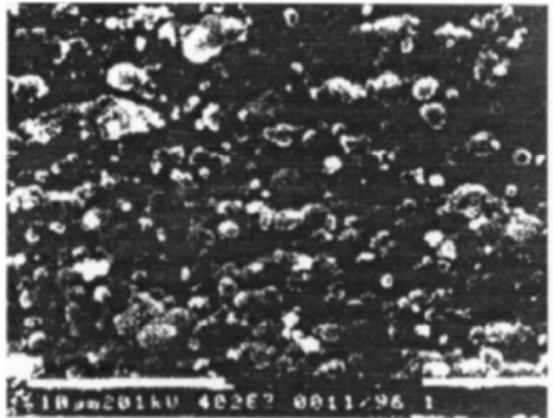

SEM (4000x): insonated at $800 \mathrm{kHz}$

Figure 10. Effect of $800 \mathrm{kHz}$ ultrasound on polypyrrole electrodeposition (Film produced on conducting indium-tin oxide glass, ex-acetonitrile/ 1,5 napthalenedisulphonate anion).

the incorporation of perchlorate is preferred over hexafluorophosphate, even though in the same conditions if perchlorate is not present at all then perfectly good conducting polymer films are obtained with hexafluorophosphate as the dopant anion. ${ }^{39}$ We decided to examine this unusual anion competition effect under insonation at $800 \mathrm{kHz}$, and obtained good quality electrode coatings of polypyrrole and polyazulene with only a slight decrease in the competitive effect. ${ }^{40}$ Given the ten-fold increase in limiting current of a voltammogram insonated by the same apparatus operating at this frequency ${ }^{20}$ (see Figure 4), there is a clear increase in cell hydrodynamics, but improved mass transport does not have an appreciable effect upon the composition of conducting polymer films produced during these sonicated 'competitive doping' experiments, as shown in Table 4.

Table 4. Competitive doping of poly(heterocycles) $-\mathrm{ClO}_{4}$ vs $\mathrm{PF}_{6}$ : effect of ultrasound (800 $\mathrm{KHz}$ ). All samples prepared galvanostatically at $1 \mathrm{~mA} / \mathrm{cm}^{2}$ for 1 hour on ITO-coated glass at $0.1 \mathrm{M}$ pyrrole and a total of $0.1 \mathrm{M} \mathrm{Bu}_{4} \mathrm{~N}$ electrolyte salts $(0.05 \mathrm{M}$ of each)

\begin{tabular}{ccccc}
\hline \multirow{2}{*}{ Solvent } & Monomer & \multicolumn{3}{c}{ EDAX Ratio } \\
\cline { 3 - 5 } & & Elements & Silent & Ultrasound \\
\hline Nitromethane & pyrrole & $\mathrm{Cl} / \mathrm{P}$ & 44 & 27 \\
Propylene carbonate & pyrrole & $\mathrm{Cl} / \mathrm{P}$ & 17 & 24 \\
Acetonitrile & pyrrole & $\mathrm{Cl} / \mathrm{P}$ & 10 & 5.5 \\
Water & pyrrole & $\mathrm{Cl} / \mathrm{P}$ & 2.3 & 1.9 \\
Acetonitrile & azulene & $\mathrm{Cl} / \mathrm{P}$ & 26 & 15 \\
\hline
\end{tabular}

This applies both to the easier to interpret competition between perchlorate and tetrasulphonato copper phthalocyanine, and the less-readily explained competition between perchlorate and hexafluorophosphate. However, this result confirms the ability of ultrasound to 
probe electrochemical mechanisms, because the origin of the competitive doping effect is a stage in the process that is little affected by insonation.

A further example of ultrasound affecting electrode coatings concerns the electroreduction of 6phenyl 2,3-dihydro-1,4-diazepinium cations (6PD). The author earlier studied these in silent conditions at St Andrews University with D.M.G. Lloyd and C.A. Vincent. When 6PD is reduced at a mercury pool cathode in dimethylformamide solvent it gives a novel pyrrolodiazepine (see Figure 11a) in high (>80\%) yield. This is a good example of the use of electrosynthesis as a methodology, because the unusual fused pyrrolodiazepine system is relatively thermally unstable, but the electrolysis is performed in a cooled cell at or below room temperature, and the product can easily be extracted in high purity by the simple addition of water to the electrolysis mixture and filtration of the resultant solid. ${ }^{41}$ The first step of the mechanism of formation of the pyrrolodiazepine is dimerisation of uncharged radicals formed by reduction of the dihydrodiazepinium cation. The dimer is not isolated because of the unsubstituted nitrogen atoms, which allow nucleophilic attack leading to internal cyclisation, with at various stages in the mechanism the loss of protons from nitrogen and expulsion of one ethylene diamine unit (in a reversal of the formation of 2,3-dihydro-1,4-diazepinium cations which are assembled by incorporation of ethylene diamine into the ring -see Figure 11b). However, if the nitrogen atoms are blocked by alkyl groups, then the internal cyclisation reaction to the pyrrolodiazepine ring does not occur and the dimer can be isolated. In the case of the 1,4 dibenzyl-2,3-dihydro-1,4-diazepinium cation (DB6PD) the total yield of products obtained from reduction in dimethylformamide at a mercury pool cathode is again $>90 \%$, but this time there is a mixture of three products, in roughly $30 \%$ yield each. ${ }^{42,43}$ There are the two stereoisomers of the dimeric bistetrahydrodiazepinyl (dl- and meso-) as expected, but there is also unexpectedly a novel tetrahydroimidazolylbutadiene, presumably obtained by a mechanism involving retrocyclisation and recyclisation processes; see Figure 12.

A feature of all this work was the use of a mercury pool cathode. This is of great benefit in electrosynthesis, because it is a heavy liquid whose surface can be stirred to avoid the build-up of electrode coatings that could eventually terminate reaction by blocking access to the electrode. This is a common problem, especially in industrial scaled-up systems where minor by-products at an electrode surface often demand the withdrawal of the fouled electrode from the cell to be mechanically abraded, with consequent disruption of the electrolysis. However, mercury is toxic, and its use is banned or soon to be banned in many countries of the world, but there is no other suitable liquid metal that has in particular a high overpotential for hydrogen evolution (and hence lack of interference from this competing reduction process). Ultrasonic vibration of a solid electrode remains a possible way to avoid fouling of a solid electrode surface, and novel electrode materials such as boron-doped diamond have been developed to mitigate the overpotential problem for 


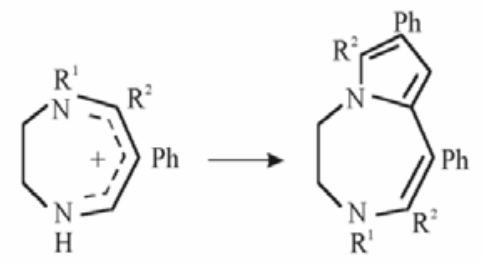

(VI)

(VII)

$$
\begin{aligned}
& a ; R^{1}=R^{2}=H \\
& b ; R^{1}=M e, R^{2}=H \\
& \text { c; } R^{1}=H, R^{2}=M e
\end{aligned}
$$

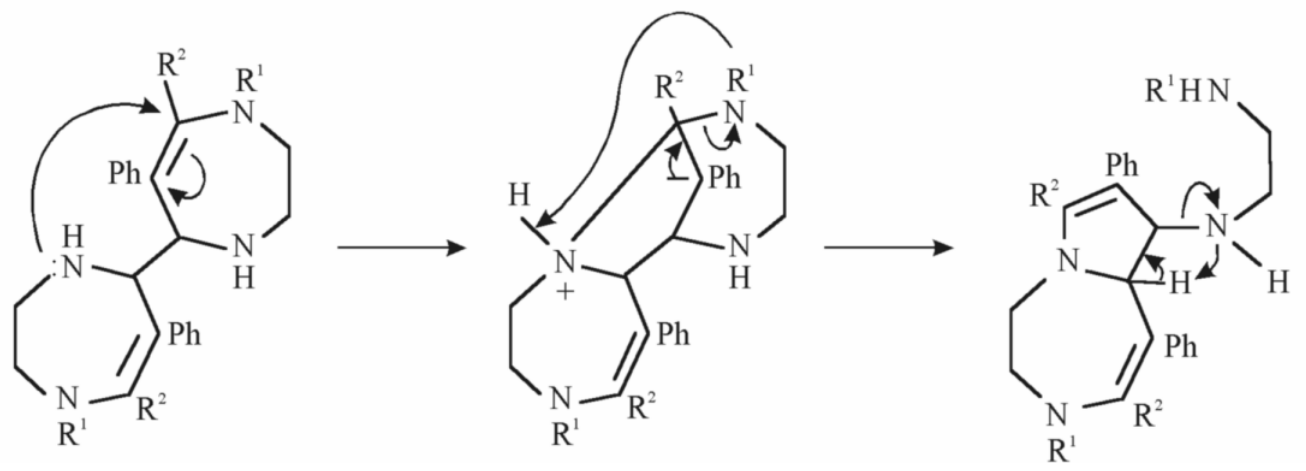

Figure 11. Electroreduction of 6-phenyl 2,3-dihydro 1,4-diazepinium perchlorates at mercury in dimethylformamide:

Upper Scheme : Overall reaction to pyrrolodiazepine

Lower Scheme: Proposed reaction mechanism after initial radical dimerisation

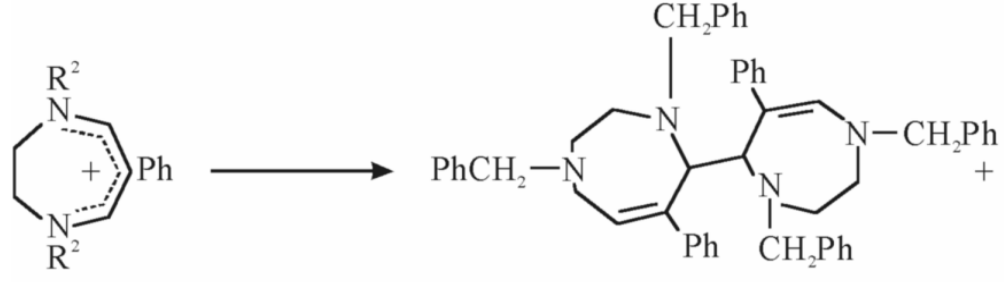

(I)
(II)<smiles>C(=C(c1ccccc1)C1N(Cc2ccccc2)CCN1Cc1ccccc1)c1ccccc1</smiles>

(III)

$\begin{array}{ll}\mathrm{R}^{1}=\mathrm{R}^{2}=\mathrm{PhCH}_{2} & \text { a; meso } \\ & \text { b; racemic }\end{array}$

Figure 12. Electroreduction of 1,4-dibenzyl 6-phenyl 2,3-dihydro 1,4-diazepinium perchlorate (I) at mercury in dimethylformamide to give bistetrahydrodiazepinyls (II) and di-imidazolidinyl butadiene (III) reductions, particularly for electroanalytical applications. This material is now becoming available for larger-area electrodes of use in laboratory-scale electrosynthesis. 
We noticed in our earlier work on dihydrodiazepinium reductions that a solid platinum electrode became fouled during the electrolysis, and so could not be used to replace mercury in silent conditions, and in any case seeking reactions that offer a choice of products, and noting particularly the unusual tetrahydroimidazolylbutadiene product from DB6PD reduction, we chose this reaction for sonoelectrosynthetic studies. We expected insonation from a $38 \mathrm{kHz}$ cleaning bath to keep a platinum electrode surface clean, and were surprised to observe that under ultrasound the electrode became fouled more rapidly, such that after only some tens of seconds the constant-current source could not maintain the necessary cell current. In silent conditions the same source could maintain current for a few minutes. ${ }^{44}$ The effect of ultrasound upon product distribution therefore could not be studied, but this system again demonstrates the capability of sonication to influence steps in a reaction pathway, but here to enhance formation of the electrode coating.

\section{Other sonoelectrochemical studies}

There are numerous studies in this expanding field: for example other synthetic-scale reactions of interest involve studies at low temperature, ${ }^{45}$ also the degradation of pollutants and environmental remediation, ${ }^{46}$ and ultrasonic emulsification of the electrolyte to allow the use of aqueous media to obviate environmentally-damaging organic solvents. ${ }^{47}$ In electroanalysis the improvement of limiting current is per se a benefit, but other considerable enhancements include the diminishment of fouling problems in difficult media such as mud, blood, landfill leachates, or even egg-white $^{48}$ and mucus ${ }^{49}$. Other improved difficult analyses are DNA bases, ${ }^{50}$ and vitamin C. ${ }^{51}$. These are interesting because ultrasound need not necessarily be applied throughout the electroanalytical measurement; and a combination of different electrochemical and sonochemical pulsing regimes can give additional benefit. Another use of differential timebase for perturbations is in the production of metal, metal oxide or semiconductor nanopowders by employing the electrically-insulated tip of an ultrasonic horn directly as the electrode ('sonotrode') and alternating sonic and electrode potential pulses with an intervening 'both off' cycle. The nanopowders can be used onwards for a variety of purposes, including in chemical synthesis. $^{52}$

The replacement of mercury has prompted improved anodic stripping for metals and adsorptive stripping for organic compounds and other species, by use of ultrasound at solid electrodes. The sound may be employed at the pre-concentration phase, or the stripping phase, or both, allowing further opportunities for manipulation of parameters. Many analyses have been examined, of which metals in media such as wine ${ }^{53}$ or soft drinks ${ }^{54}$ are just two examples. The Oxford group has also widely studied the use of boron-doped diamond as an ultrasonically-stable

electrode material with a wide cathodic window. ${ }^{55}$ Other areas of interest in sonoelectrochemistry include corrosion, batteries, photoelectrochemistry, bioelectrochemistry and sensors. 


\section{Summary}

Insonation of electrochemical systems produces a variety of interesting and beneficial effects and can act as a probe of reaction mechanism. There is increasing interest in this methodology, and there is not room to discuss all aspects or acknowledge all developments made by the many workers currently experimenting in this exciting field. In addition there are other multiperturbation techniques in electrochemistry that might be considered as 'extreme or nonclassic' in the European Union initiative. These include the use of simultaneous microwave irradiation, temperature-jump and high-speed non-equilibrium thermal excursions, very low temperatures, high pressures and supercritical fluid electrolytes, or magnetic field effects or centrifugal and gravitational excursions. All of these have potential benefits in various aspects of electrochemistry, and these multiperturbation methodologies show that the discipline has a promising future.

\section{Acknowledgements}

The author thanks D.M.G. Lloyd, C.A. Vincent, J.H.P. Utley, J.M. Mellor, A. Bewick, and D.R. Rosseinsky for variously exciting and maintaining his interest in electrochemistry; also members of the European Union Human Capital Network and COST D10 Working Group, also T.J. Mason, J.P. Lorimer, S.S. Phull of the Coventry Sonochemistry Centre, I.R. Peterson and J. Heptinstall of the Coventry Centre for Molecular and Biomolecular Electronics, G. Elliott, R. Lines, R.J. Mortimer and many others. Particular thanks go to R.G. Compton.

EPSRC, NAB, the European Union and Coventry University are thanked for financial support.

\section{References}

1. For reviews of electroorganic chemistry see for example: (a) Baizer, M.M.; Lund, H. Eds. Organic Electrochemistry; $3^{\text {rd }}$ Edn. Marcel Dekker; (b) Fry, A.J. Synthetic Organic Electrochemistry, Harper and Row, 1972.

2. Moriguchi. N. J. Chem. Soc. Japan 1934, 5, 751.

3. See Yeager, E. Modern Aspects of Electrochemistry; Bockris, J.O.'M.; Conway, B.E.; White, R.E.. Eds.; Plenum: New York, Chapter 1, 1979.

4. Walker, R. In Advances in Sonochemistry; Mason T.J. Ed.; JAI Press, 1993; Vol. 3.

5. Connors, T.F.; Rusling, J.F. Chemosphere 1984, 13, 415.

6. See for example Price, G.J. Ed. Current Trends in Sonochemistry; RSC Publications, 1992.

7. Toppare, L.; Akbulut, U.; Yurrtas, B. Polymer, 1986, 27, 803 and other papers in this series.

8. Gautheron, B; Degrand, C.; Tainturer, G. J. Am. Chem. Soc. 1985, 197, 5579.

9. Walton, D.J.; Chyla, A.; Lorimer, J.P.; Mason, T.J.; Smith, G. Chem. Comm. 1989, 603. 
10. Osawa, S.; Ito, M.; Tanaka, K.; Kuwano, J. Synthetic Metals 1987, 18, 145.

11. Ono, Y.; Nishiki, Y.; Nonaka ,T. Chem. Lett. 1994, 1623.

12. Walton, D.J.; Phull, S.S. In Advances in Sonochemistry; Mason, T.J., Ed.; JAI Press, 1996, Vol. 4 and references therein.

13. Walton, D.J.; Mason, T.J. In Synthetic Organic Sonochemistry; Luche, J.-L., Ed.; Plenum Press: New York, 1998 and references therein.

14. Atobe, M.; Nonaka, T.; Nippon Kagaku Kaishi 1998, 219.

15. Saterlay, A.J.; Compton, R.G.; Fresenius, J. Anal. Chem. 2000, 367, 308 and references therein.

16. Compton, R.G.; Eklund, J.C.; Marken, F. Electroanalysis 1997, 9, 509 and references therein.

17. (a) EU Human Capital and Mobility Network Grant ERBCHRX 940475 Ultrasonic Modification of Electroorganic Processes. (b) EU COST Working Group D10/0010/98 Sonoelectrochemical Processes.

18. Mason, T.J.; Lorimer, J.P. Sonochemistry, Ellis Horwood ,1989.

19. See for example Suslick, K.S. Ed. Ultrasound: its Chemical, Physical and Biological Effects; Verlag, Chemie 1988.

20. Walton, D.J.; Phull, S.S.; Chyla, A.; Lorimer, J.P.; Mason, T.J.; Burke, L.D.; Murphy, M.; Compton, R.G.; Eklund, J.C.; Page, S.D. J. Applied Electrochem. 1995, 25, 1083.

21. Eklund, J.C.; Marken, F.; Waller, D.N.; Compton, R.G. Electrochimica Acta 1996, 41, 1541.

22. Birkin, P.R.; Silva-Martinez, S. J. Electroanal. Chem. 1996, 416, 127.

23. Atobe, M.; Tonoi, T.; Nonaka, T. Electrochem. Comm. 1999, 1, 593.

24. Atobe, M.; Nonaka, T. Chemistry Lett. 1997, 323.

25. Nyberg, K. Chem. Commun. 1969, 774.

26. Utley, J.H.P.; Eberson, L. In Organic Electrochemistry $3^{\text {rd }}$ Edn; Baizer, M.M.; Lund, H., Eds.; Marcel Dekker: New York, 1991.

27. Walton, D.J. Chyla, A.; Mason, T.J.; Lorimer, J.P. Synth. Commun. 1990, 20, 1843.

28. Fujiwara, H.; Atobe, M.; Kanetsuna, H.; Nonaka, T. J. Chinese Chem. Soc. 1998, 45, 175.

29. Wadhawan, J.D.; Del Campo, F.J.; Compton, R.G.; Foord, J.S.; Marken, F.; Bull, S.D. Davies SG, Walton DJ, Ryley S, J. Electroanal. Chem. 2001, 507, 135.

30. Walton D.J.; Phull S.S.; Geissler U.; Chyla A.; Durham A.; Ryley S.; Mason T.J; Lorimer J.P. Electrochem. Commun. 2000, 2, 431.

31. Koehl, W.J. Jr. J. Org. Chem. 1967, 32, 614.

32. Compton, R.G.; Eklund, J.C.; Page, S.G.; Sanders, G.H.W.; Booth, J. J. Phys. Chem. 1994, 98, 12410.

33. Walton, D.J.; Phull, S.S.; Bates, D.M.; Lorimer, J.P.; Mason, T.J. Electrochimica Acta 1993, $18,307$.

34. Malins, C.; Vandeloise, R.; Walton, D.; Vanderdonckt, E. J. Phys Chem. 1997, 101, 5063.

35. Atobe, M.; Kaburagi, T.; Nonaka, T. Electrochemistry 1999, 67, 1114. 
36. Walton, D.J.; Geissler, U.; Chyla, A.; Mason, T.J.; Lorimer, J.P. Proceedings of World Congress on Ultrasonics, Berlin 1996.

37. Walton, D.J.; Hadingham, D.; Viney, I.V.F.; Hall, C.E.; Chyla, A. Synth. Met. 1991, 43, 363.

38. Walton, D.J.; Viney, I.V.F.; Mure J.-M.; Hall, C.E.; Chyla, A. Synth. Met. 1993, 55, 1465.

39. Walton, D.J.; Viney, I.V.F.; Ryley, S.; Taylor, E. Advanced Materials for Optics and Electronics 1996, 6, 395.

40. Walton, D.J.; Chyla, A; Ryley, S; Geissler, U. manuscript in preparation.

41. (a) Loyd, D.M.G.; Vincent, C.A.; Walton, D.J.; Declerq, J.-P.; Germain, G.; Van Meerssche, M. Chem. Commun. 1978, 499. (b) Lloyd, D.M.G.; Walton, D.J.; Nijns, C.; Vincent, C.A. J. Chem. Soc. Perkin Trans. 2 1980, 1441.

42. Lloyd, D.M.G.; Vincent. C.A.; Walton, D.J.; Declerq, J.-P.; Germain, G.; Van Meerssche, M. Bull Soc. Chim. Belges 1979, 88, 113.

43. Lloyd, D.M.G.; Vincent, C.A.; Walton, D.J. J.Chem.Soc., Perkin Trans. 2 1981, 801.

44. Walton, D.J.; Uzzell, G; Clarke, L. unpublished results.

45. Del Campo, F.J.; Maisonhaute, E.; Compton, R.G.; Marken, F.; Aldaz, A. J. Electroanal. Chem. 2001, 506, 170.

46. Lorimer, J.P.; Mason, T.J.; Plattes, M.; Phull, S.S. Ultrasonics Sonochemistry 2000, 7, 237 this reference refers to azo dye pollutants but there are many other example.

47. Marken, F.; Compton, R.G. Electrochimica Acta 1998, 43, 2157.

48. Davis, J; Compton, R.G. Analytica Chimica Acta 2000, 404, 241.

49. Hardcastle, J. L.; Compton, R.G. Electroanalysis 2001, 13, 89.

50. Oliveira-Brett, A.M.; Matysik, F.M. J. Electroanal. Chem. 1997, 429, 95.

51. Compton, R.G. Matysik, F.M. Electroanalysis 1996, 8, 218.

52. Durant, A.; Delplancke, J.L.; Libert, V.; Reisse, J. Eur. J. Org. Chem. 1999, 2845 and other papers in this series.

53. Akkermans, R.P.; Ball, J.C.; Rebbitt, T.G.; Compton, R.G. Electrochim. Acta 1998, 43, 3443.

54. Akkermans, R.P.; Wu, M.; Compton, R.G. Electroanalysis 1998, 10, 814.

55. Saterlay, A.J.; Marken, F.; Foord, J.S.; Compton, R.G. Talanta 2000, 53, 403. 